\title{
Focused Section on Matrix Computations
}

\author{
Zhong-Zhi Bai ${ }^{1}$
}

Published online: 16 October 2020

(c) Shanghai University 2020

During April 19-23, 2019, the International Workshop on Matrix Computations - Gene Golub Memorial Day 2019, was held at Lanzhou University, Lanzhou, China. About 60 senior researchers, young scholars, and graduate students attended this workshop, including several famous numerical linear algebraists such as Michele Benzi from Italy, Walter Gander and Martin H. Gutknecht from Switzerland, Wai-Ki Ching and Franklin T. Luk from Hong Kong, China, and Yu-Jiang Wu from the mainland of China. On the workshop, the winners of the "Gene Golub Memorial Workshop Best Presentation Prize" were ZengQi Wang from Shanghai Jiao Tong University (for the best oral presentation) and Jun-Feng Yin from Tongji University (for the best poster presentation), both from Shanghai, China. Each of the winners received a plaque, a certificate, and some kind of gifts.

The two organizers of the workshop are Zhong-Zhi Bai (Chinese Academy of Sciences) and Yu-Mei Huang (Lanzhou University), and the two main financial sponsors are Lanzhou University and Tianyuan Mathematical Center in Northwest China.

The participants strongly believed that it is necessary to formally publish some of the high-quality presentations to record this meaningful and valuable academic activity. So, after consulting with the editor-in-chief, Prof. Chi-Wang Shu, we are very glad that he approved our proposal of publication on the journal Communications on Applied Mathematics and Computation. This focused section consists of six regularly reviewed papers, which were elaborately selected from submissions of some of the presenters of the workshop. The content of these papers covers iterative solutions of Hermitian eigenvalue problems and linear matrix equations, structured preconditioners for saddle-point linear systems and discretized space-fractional diffusion equations, modulus-based matrix splitting iteration methods for linear complementarity problems, and randomized generalized singular value decompositions. We thank all authors of these contributions, and hope that the readers are interested in the topics, techniques and methods, and results of these works.

Last but not the least, we thank Profs. Chi-Wang Shu and He-Ping Ma, as well as Yi Ding and Chun-Zhi Pan, for their great help during the submission, review, and publication processes of this focused section.

Zhong-Zhi Bai

bzz@1sec.cc.ac.cn

1 Institute of Computational Mathematics, Academy of Mathematics and Systems Science, Chinese Academy of Sciences, Beijing 100190, China 